\title{
Convergent synthesis of the tetrasaccharide repeating unit of the 0 -antigen of Shigella boydii type 9
}

\author{
Abhishek Santra and Anup Kumar Misra*
}

\author{
Full Research Paper \\ Address: \\ Bose Institute, Division of Molecular Medicine, P-1/12, C.I.T. Scheme \\ VII-M, Kolkata-700054, India; Fax: 91-33-2355 3886 \\ Email: \\ Anup Kumar Misra* - akmisra69@gmail.com \\ * Corresponding author \\ Keywords: \\ diarrhea; glycosylation; O-antigen; oligosaccharide; Shigella boydii
}

\author{
Beilstein J. Org. Chem. 2011, 7, 1182-1188. \\ doi:10.3762/bjoc.7.137 \\ Received: 01 June 2011 \\ Accepted: 25 July 2011 \\ Published: 29 August 2011 \\ Associate Editor: S. Flitsch \\ (C) 2011 Santra and Misra; licensee Beilstein-Institut. \\ License and terms: see end of document.
}

\begin{abstract}
A convenient synthesis of the tetrasaccharide repeating unit of the $O$-antigen of Shigella boydii type 9 has been achieved in excellent yield using a [2 + 2] block glycosylation strategy. TEMPO-mediated selective oxidation of the primary alcohol of the tetrasaccharide derivative $\mathbf{8}$ to the carboxylic group followed by deprotection of the functional groups furnished target tetrasaccharide $\mathbf{1}$ as its 4-methoxyphenyl glycoside in high yield.
\end{abstract}

\section{Introduction}

Diarrhoeal disease is a common cause of death in the tropical countries and it is the second mostly causing infant deaths worldwide. Shigella is one of the well-studied human pathogens that cause diarrhoeal disease and dysentery (e.g., shigellosis). Among several types of Shigella species, Shigella dysenteriae is the most virulent pathogen causing devastating health problems in developing countries [1-3]. Shigella strains are classified into four species: Shigella boydii, Shigella dysenteriae, Shigella flexneri and Shigella sonnei [4]. Sometimes, these species are also termed as Shigella subgroups A, B, C, and D. Based on the $O$-antigens, the Shigella species are divided into multiple serotypes [5]. In general, $O$-antigens of Shigella species are acidic in nature because of the presence of acidic constituents (e.g., uronic acid, pseudaminic acid etc. or lactic acid, pyruvic acid etc.) in their structures [6,7]. Recently, L'vov et al. reported the structure of the $O$-antigen of Shigella boydii type 9, which is a tetrasaccharide repeating unit containing a D-glucuronic acid moiety (Figure 1) [8].

\section{$\rightarrow 3)-\alpha-D-G l c p N A c-(1 \rightarrow 3)-\alpha-L-R h a p-(1 \rightarrow 4)-\alpha-D-G l c p-(1 \rightarrow 4)-\beta-D-G l c p A-(1 \rightarrow$}


Development of effective therapeutics to control the infections of drug-resistant bacterial strains is the thrust area in the medicinal chemistry. Like other bacterial infections, emergence of the drug resistant Shigella infections requires development of the newer therapeutics than the earlier used anti-shigellosis agents $[9,10]$. Because of the high antigenic nature of the $O$-antigens, antibodies against the $O$-specific polysaccharide of a particular Shigella strain have the potential to control Shigella infections [11-14]. A number of reports have been cited earlier to develop glycoconjugate based therapy to control Shigella infections [15-19]. In order to develop a glycoconjugate based therapeutic agent from the tetrasaccharide repeating unit of the $O$-antigen of Shigella boydii type 9, it is essential to perform several immunochemical studies with the glycoconjugates derived from this tetrasaccharide repeating unit. The large quantity of the tetrasaccharide that is required for this purpose cannot be accessible from a natural source. Therefore, chemical synthesis is the only option for achieving this in large quantity. As a first step towards the preparation of glycoconjugates, we report herein a convergent chemical synthesis of the tetrasaccharide as its 4-methoxyphenyl glycoside $\mathbf{1}$ corresponding to the $O$-antigen of Shigella boydii type 9 using a [2+2] block glycosylation strategy (Figure 2).

\section{Results and Discussion}

The convergent synthesis of the target tetrasaccharide $\mathbf{1}$ as its 4-methoxyphenyl glycoside has been achieved applying a number of recently developed elegant reaction methodologies. A number of notable features are present in the synthetic strategy, which are (a) convergent [2+2] block glycosylation; (b) application of recently developed environmentally benign reaction conditions for protecting group manipulations and glycosylations such as, (i) $O$-acetylation using sulfamic acid [20], (ii) regioselective ring opening of the benzylidene acetal using a combination of triethylsilane and iodine [21], (iii) direct one-pot conversion of $O$-acetyl group to $O$-benzyl group [22], (iv) activation of glycosyl trichloroacetimidate and thioglycoside donors by perchloric acid supported on silica $\left(\mathrm{HClO}_{4}-\mathrm{SiO}_{2}\right)$ [23-26], and late stage TEMPO mediated selective oxidation [27-29] of the primary hydroxy group to the carboxylic group under phase transfer conditions without affecting the secondary hydroxy groups; (c) use of 4-methoxyphenyl (PMP) group as anomeric protecting group [30,31], which can be easily removed under oxidative conditions for the preparation of glycoconjugate derivatives. The synthesis of the target tetrasaccharide $\mathbf{1}$ was achieved by the stereoselective coupling of a D-maltose derived disaccharide derivative $\mathbf{2}$ and a disaccharide thioglycoside derivative $\mathbf{7}$ followed by functional group manipulations of the resulting tetrasaccharide derivative 8. For this purpose, suitably functionalized reaction intermediates 2, 3, 4 and 7 were prepared from commercially available reducing mono- and disaccharides utilizing a series of reaction methodologies reported earlier.

4-Methoxyphenyl (4,6- $O$-benzylidene- $\alpha$-D-glucopyranosyl)$(1 \rightarrow 4)-\beta$-D-glucopyranoside (5) [32] was subjected to a sequence of reactions involving acetylation using acetic anhydride in the presence of sulfamic acid [20] followed by regioselective reductive ring opening of the benzylidene acetal using a combination of triethylsilane and iodine [21] to furnish 4-methoxyphenyl (2,3-di- $O$-acetyl-6- $O$-benzyl- $\alpha$-D-glucopyranosyl)-( $1 \rightarrow 4)$-2,3,6-tri- $O$-acetyl- $\beta$-D-glucopyranoside $(2)$ in $82 \%$ yield (Scheme 1). 3,4,6-Tri- $O$-acetyl-2-azido-2-deoxy- $\alpha$ D-glucopyranosyl trichloroacetimidate (3) [33] was allowed to couple stereoselectively with ethyl 2,4-di- $O$-benzyl-1-thio- $\alpha$-Lrhamnopyranoside (4) [34] under Schmidt's reaction conditions [35] using perchloric acid supported on silica $\left(\mathrm{HClO}_{4}-\mathrm{SiO}_{2}\right)$ $[23,24]$ as glycosylation activator to give ethyl $(3,4,6$-tri- $O$ acetyl-2-azido-2-deoxy- $\alpha$-D-glucopyranosyl)-(1 $\rightarrow 3)-2,4$-di- $O$ benzyl-1-thio- $\alpha$-L-rhamnopyranoside (6) in $81 \%$ yield. Stereo-
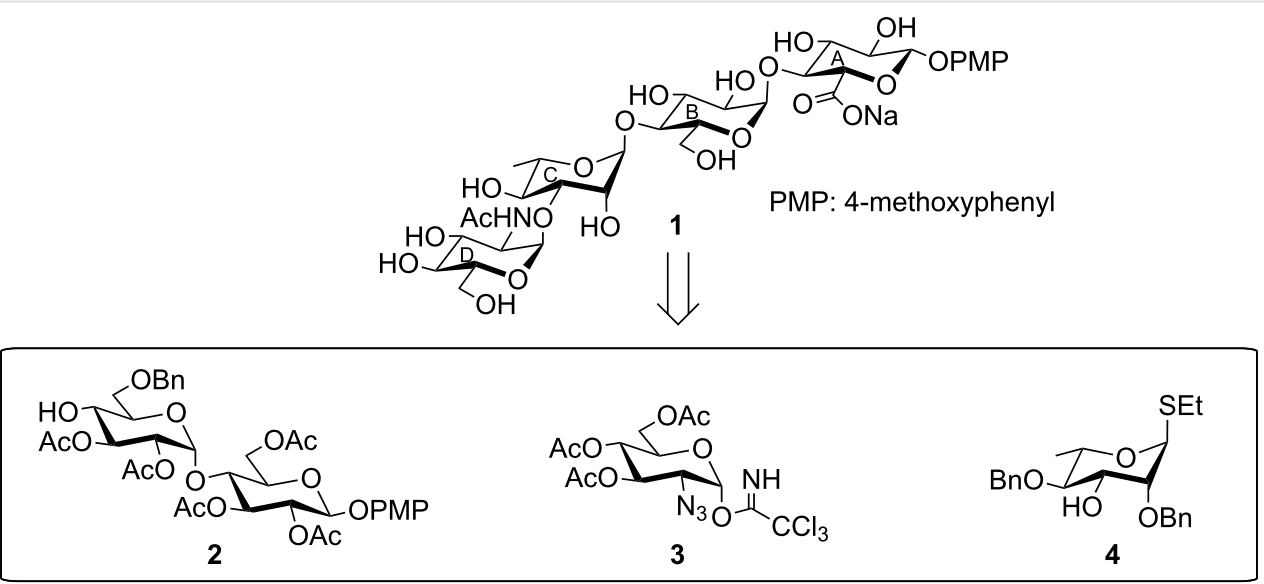

Figure 2: Structure of the synthesized tetrasaccharide and its intermediates. 


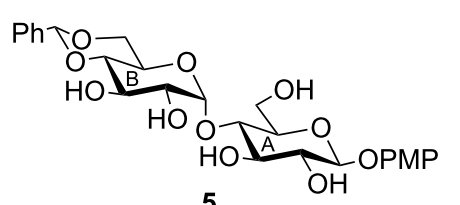

5

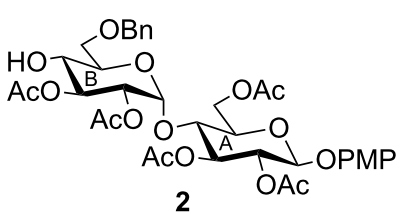

Scheme 1: Reagents: (a) acetic anhydride, sulfamic acid, $60{ }^{\circ} \mathrm{C}, 30 \mathrm{~min}, 91 \%$; (b) $\mathrm{Et}_{3} \mathrm{SiH}, \mathrm{I}_{2}, 0-5{ }^{\circ} \mathrm{C}, 30 \mathrm{~min}, 82 \%$

selective formation of compound $\mathbf{6}$ was confirmed from its spectral analysis (presence of signals at $\delta 5.35$ (br s, H-1 $\mathrm{C}$ ), 4.95 $\left(\mathrm{d}, J=3.6 \mathrm{~Hz}, \mathrm{H}-1_{\mathrm{D}}\right)$ in the ${ }^{1} \mathrm{H} \mathrm{NMR}$ and signals at $\delta 92.8$ $\left(\mathrm{C}-1_{\mathrm{D}}\right), 81.0\left(\mathrm{C}-1_{\mathrm{C}}\right)$ in the ${ }^{13} \mathrm{C}$ NMR spectrum $)$. Compound 6 was transformed into disaccharide thioglycoside donor 7 in $91 \%$ yield under a one-pot deacetylation-benzylation reaction condition [22] (Scheme 2). In this case, the thioethyl group acts as an orthogonal anomeric protecting group since it acts as a glycosyl acceptor in the case of compound $\mathbf{4}$ whereas compound $\mathbf{7}$ has been used as the glycosyl donor in the next step. Iodonium ion promoted stereoselective glycosylation of the disaccharide thioglycoside donor $\mathbf{7}$ with the disaccharide acceptor $\mathbf{2}$ in the presence of a combination of $\mathrm{N}$-iodosuccinimide (NIS) and $\mathrm{HClO}_{4}-\mathrm{SiO}_{2}[25,26]$ furnished tetrasaccharide derivative 8 in $82 \%$ yield. Stereoselective formation of new $\alpha$-glycosyl linkage in compound 8 was confirmed from the 1D and 2D NMR spectral analysis (presence of signals at $\delta 5.34\left(\mathrm{~d}, J=4.5 \mathrm{~Hz}, \mathrm{H}-1_{\mathrm{B}}\right)$, 4.97 (d, $\left.J=3.5 \mathrm{~Hz}, \mathrm{H}-1_{\mathrm{D}}\right), 4.95$ (d, $\left.J=8.0 \mathrm{~Hz}, \mathrm{H}-1_{\mathrm{A}}\right), 4.89$ (d, $\left.J=2.0 \mathrm{~Hz}, \mathrm{H}-1_{\mathrm{C}}\right)$ in the ${ }^{1} \mathrm{H}$ NMR and signals at $\delta 99.7\left(\mathrm{C}-1_{\mathrm{A}}\right)$, $98.8\left(\mathrm{C}-1_{\mathrm{C}}\right), 96.0\left(\mathrm{C}-1_{\mathrm{B}}\right), 93.9\left(\mathrm{C}-1_{\mathrm{D}}\right)$ in the ${ }^{13} \mathrm{C}$ NMR spectrum). Because of the presence of the non-participating benzyl- oxy group in the C-2 position of the L-rhamnosyl moiety, involved in the glycosylation reaction, a minor amount of $\beta$-glycosyl linked product also formed $(\sim 6 \%)$ together with the desired product $\mathbf{8}$, which was separated by the column chromatography. The presence of the $\alpha$-linkages in compound $\mathbf{8}$ was further confirmed from the $J_{\mathrm{C} 1-\mathrm{H} 1}$ values in the ${ }^{1} \mathrm{H}$ coupled ${ }^{13} \mathrm{C}$ NMR spectrum of compound 8 . Appearance of $J_{\mathrm{C} 1-\mathrm{H} 1}$ $172.0 \mathrm{~Hz}, 171.6 \mathrm{~Hz}, 170.5 \mathrm{~Hz}$ and $162.0 \mathrm{~Hz}$ values in the anomeric region in the ${ }^{1} \mathrm{H}$ coupled ${ }^{13} \mathrm{C}$ spectrum unambiguously supported the presence of three $\alpha$-linkages and one $\beta$-linkage [36-38] in compound $\mathbf{8}$. Compound $\mathbf{8}$ was subjected to a reaction sequence involving (a) deacetylation using $0.1 \mathrm{M}$ sodium methoxide in methanol; (b) TEMPO mediated selective oxidation [27-29] of the primary hydroxy group leaving secondary hydroxy groups unaffected in a phase transfer reaction condition and (c) removal of benzyl groups for cleavage of ethers and reduction of the azido group to an amine by hydrogenation over $20 \% \mathrm{Pd}(\mathrm{OH})_{2} / \mathrm{C}$ followed by $\mathrm{N}$-acetylation to furnish target tetrasaccharide $\mathbf{1}$ as its sodium salt and 4-methoxyphenyl glycoside in $64 \%$ yield (Scheme 3 ). Spectroscopic analysis of compound 1 confirmed its formation (presence of signals at $\delta 5.20$

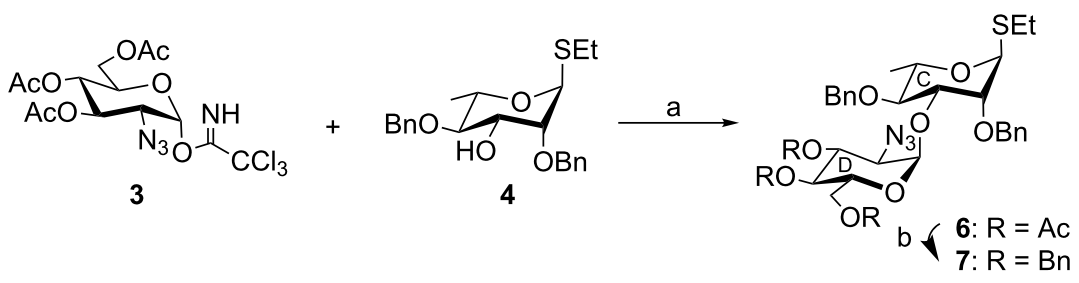

Scheme 2: Reagents: (a) $\mathrm{HClO}_{4}-\mathrm{SiO}_{2}, \mathrm{CH}_{2} \mathrm{Cl}_{2},-15^{\circ} \mathrm{C}, 1 \mathrm{~h}, 81 \%$; (b) benzyl bromide, $\mathrm{NaOH}, n-\mathrm{Bu}_{4} \mathrm{NBr}, \mathrm{THF}, \mathrm{rt}, 2 \mathrm{~h}, 91 \%$.

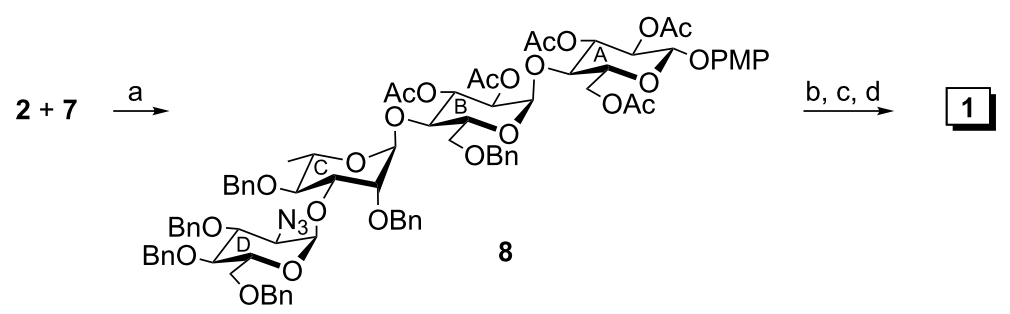

Scheme 3: Reagents: (a) $\mathrm{N}$-iodosuccinimide, $\mathrm{HClO}_{4}-\mathrm{SiO}_{2},-10{ }^{\circ} \mathrm{C}, 1 \mathrm{~h}, 82 \%$; (b) $0.1 \mathrm{M} \mathrm{CH}_{3} \mathrm{ONa}, \mathrm{CH}_{3} \mathrm{OH}, \mathrm{rt}, 3 \mathrm{~h}$; (c) (i) $\mathrm{NaBr}$, TBAB, TEMPO, $\mathrm{CH}_{2} \mathrm{Cl}_{2}, \mathrm{H}_{2} \mathrm{O}, \mathrm{NaOCl}, \mathrm{NaHCO}_{3}, 5^{\circ} \mathrm{C}, 2 \mathrm{~h}$; (ii) tert-butanol, 2-methylbut-2-ene, $\mathrm{NaClO}_{2}, \mathrm{NaH}_{2} \mathrm{PO}_{4}, \mathrm{rt}, 3 \mathrm{~h}$; (d) (i) $\mathrm{H}_{2}, 20 \% \mathrm{Pd}(\mathrm{OH})_{2} / \mathrm{C}, \mathrm{CH}{ }_{3} \mathrm{OH}-\mathrm{EtOAc}$, rt, $10 \mathrm{~h}$; (ii) acetic anhydride, $\mathrm{CH}_{3} \mathrm{OH}$, rt, 30 min, overall $64 \%$. 
(d, $\left.J=3.6 \mathrm{~Hz}, \mathrm{H}-1_{\mathrm{B}}\right), 4.93$ (br s, $\left.\mathrm{H}-1_{\mathrm{D}}\right), 4.89$ (br s, $\mathrm{H}-1_{\mathrm{C}}$ ), 4.82 $\left(\mathrm{d}, J=7.8 \mathrm{~Hz}, \mathrm{H}-1_{\mathrm{A}}\right)$ in the ${ }^{1} \mathrm{H}$ NMR and signals at $\delta 103.3$ $\left(\mathrm{C}-1_{\mathrm{A}}\right), 102.8\left(\mathrm{C}-1_{\mathrm{B}}\right), 102.4\left(\mathrm{C}-1_{\mathrm{C}}\right), 96.7\left(\mathrm{C}-1_{\mathrm{D}}\right)$ in the ${ }^{13} \mathrm{C}$ NMR spectrum).

\section{Conclusion}

In conclusion, a convenient synthetic strategy has been developed for the synthesis of the tetrasaccharide repeating unit of the $O$-antigen of Shigella boydii type 9 as its 4-methoxyphenyl glycoside sodium salt using a [2+2] block synthetic strategy. Use of a block glycosylation strategy and a late-stage selective oxidation of the primary hydroxy group significantly reduced the number of protection-deprotection steps. A number of modified clean reaction methodologies have been applied for the preparation of intermediates. $\mathrm{HClO}_{4}-\mathrm{SiO}_{2}$ has been used as an effective acid catalyst to activate glycosyl trichloroacetimidate derivative and thioglycoside in combination with NIS avoiding the use of moisture sensitive protic acids. All intermediate steps were high yielding and glycosylation steps were stereoselective.

\section{Experimental}

General methods: All reactions were monitored by thin layer chromatography over silica gel-coated TLC plates. The spots on TLC plates were visualized by warming ceric sulfate $(2 \%$ $\mathrm{Ce}\left(\mathrm{SO}_{4}\right)_{2}$ in $2 \mathrm{~N} \mathrm{H}_{2} \mathrm{SO}_{4}$ )-sprayed plates on a hot plate. Silica gel 230-400 mesh was used for column chromatography. ${ }^{1} \mathrm{H}$ and ${ }^{13} \mathrm{C}$ NMR, DEPT 135, 2D COSY, HMQC and gated ${ }^{1} \mathrm{H}$ coupled ${ }^{13} \mathrm{C}$ NMR spectra were recorded on Bruker Avance DRX 500 and $600 \mathrm{MHz}$ spectrometers using $\mathrm{CDCl}_{3}$ and $\mathrm{CD}_{3} \mathrm{OD}$ as solvents and TMS as internal standard unless otherwise stated. Chemical shifts are expressed in $\delta$ ppm. ESIMS were recorded on a Micromass Quattro mass spectrometer. Elementary analysis was carried out on Carlo Erba-1108 analyzer. Optical rotations were measured at $25^{\circ} \mathrm{C}$ on a Jasco P-2000 polarimeter. Commercially available grades of organic solvents of adequate purity are used in all reactions. Silica supported perchloric acid $\left(\mathrm{HClO}_{4}-\mathrm{SiO}_{2}\right)$ was prepared following the earlier report [39].

4-Methoxyphenyl (2,3-di- $O$-acetyl-6- $O$-benzyl- $\alpha$-D-glucopyranosyl)-( $\rightarrow 4)-2,3,6$-tri- $O$-acetyl- $\beta$-D-glucopyranoside (2): To a suspension of 4-methoxyphenyl (4,6-O-benzylidene- $\alpha$-Dglucopyranosyl)-( $1 \rightarrow 4)-\beta$-D-glucopyranoside $(5,3.0 \mathrm{~g}$, $5.59 \mathrm{mmol})$ in acetic anhydride $(6.0 \mathrm{~mL}, 63.47 \mathrm{mmol})$ sulfamic acid $(100 \mathrm{mg}, 1.0 \mathrm{mmol})$ was added and the reaction mixture was allowed to stir at $60{ }^{\circ} \mathrm{C}$ for $30 \mathrm{~min}$. The solvents were removed under reduced pressure and the crude reaction mixture was passed through a short pad of silica gel using hexane-EtOAc (1:2) as eluant to give pure acetylated product $(3.8 \mathrm{~g}, 91 \%)$. To a solution of the acetylated product $(3.5 \mathrm{~g}$,
$4.69 \mathrm{mmol})$ in $\mathrm{CH}_{3} \mathrm{CN}(15 \mathrm{~mL})$ triethylsilane $(1.5 \mathrm{~mL}$, $9.39 \mathrm{mmol})$ and iodine $(250 \mathrm{mg}, 0.98 \mathrm{mmol})$ were added at $0-5{ }^{\circ} \mathrm{C}$ and the reaction mixture was allowed to stir at the same temperature for $30 \mathrm{~min}$. The reaction mixture was poured into water and extracted with $\mathrm{CH}_{2} \mathrm{Cl}_{2}(100 \mathrm{~mL})$. The organic layer was successively washed with satd. $\mathrm{NaHCO}_{3}$ solution and water, dried $\left(\mathrm{Na}_{2} \mathrm{SO}_{4}\right)$ and concentrated. The crude product was purified over silica gel using hexane-EtOAc (2:1) as eluant to give pure compound 2 (2.9 g, 82\%). White solid; mp $60-62{ }^{\circ} \mathrm{C}$; $[\alpha]_{\mathrm{D}}{ }^{25}+43.2\left(c 1.2, \mathrm{CHCl}_{3}\right)$; IR (KBr): $3479,1751,1509,1371$, 1233, 1047, 830, $754 \mathrm{~cm}^{-1} ;{ }^{1} \mathrm{H} \mathrm{NMR}\left(500 \mathrm{MHz}, \mathrm{CDCl}_{3}\right) \delta 5.38$ $\left(\mathrm{d}, J=4.0 \mathrm{~Hz}, 1 \mathrm{H}, \mathrm{H}-1_{\mathrm{B}}\right), 5.28\left(\mathrm{t}, J=9.0 \mathrm{~Hz}, 1 \mathrm{H}, \mathrm{H}-3_{\mathrm{A}}\right), 5.21$ ( $\left.\mathrm{t}, J=9.0 \mathrm{~Hz}, 1 \mathrm{H}, \mathrm{H}-3_{\mathrm{B}}\right), 5.04\left(\mathrm{t}, J=8.5 \mathrm{~Hz}, 1 \mathrm{H}, \mathrm{H}-2_{\mathrm{A}}\right), 4.96$ $\left(\mathrm{d}, J=8.0 \mathrm{~Hz}, 1 \mathrm{H}, \mathrm{H}-1_{\mathrm{A}}\right), 4.80\left(\mathrm{dd}, J=9.0,4.0 \mathrm{~Hz}, 1 \mathrm{H}, \mathrm{H}-2_{\mathrm{B}}\right)$, $4.60-4.54\left(\mathrm{~m}, 2 \mathrm{H}, \mathrm{PhCH}_{2}\right), 4.50\left(\mathrm{~d}, J=11.5 \mathrm{~Hz}, 1 \mathrm{H}, \mathrm{H}-6_{\mathrm{aA}}\right)$, $4.22\left(\mathrm{dd}, J=12.0 \mathrm{~Hz}, 5.0 \mathrm{~Hz}, 1 \mathrm{H}, \mathrm{H}-6_{\mathrm{bA}}\right), 4.06$ (t, $J=9.5 \mathrm{~Hz}$, $\left.1 \mathrm{H}, \mathrm{H}-4_{\mathrm{A}}\right), 3.79-3.71\left(\mathrm{~m}, 4 \mathrm{H}, \mathrm{H}-4_{\mathrm{B}}, \mathrm{H}-5_{\mathrm{A}}, \mathrm{H}-5_{\mathrm{B}}, \mathrm{H}-6_{\mathrm{aB}}\right), 3.75$ $\left(\mathrm{s}, 3 \mathrm{H}, \mathrm{OCH}_{3}\right), 3.63-3.61\left(\mathrm{~m}, 1 \mathrm{H}, \mathrm{H}-6_{\mathrm{bB}}\right), 2.07,2.03,2.00,1.99$ $\left(4 \mathrm{~s}, 15 \mathrm{H}, 5 \mathrm{COCH}_{3}\right) ;{ }^{13} \mathrm{C} \mathrm{NMR}\left(125 \mathrm{MHz}, \mathrm{CDCl}_{3}\right) \delta 170.1$, 170.6, 170.5, 170.2, $169.6\left(5 \mathrm{COCH}_{3}\right), 155.6-114.5$ (Ar-C), $99.6\left(\mathrm{C}-1_{\mathrm{A}}\right), 95.7\left(\mathrm{C}-1_{\mathrm{B}}\right), 75.4\left(\mathrm{C}-3_{\mathrm{A}}\right), 73.7\left(\mathrm{PhCH}_{2}\right), 72.3$ $\left(\mathrm{C}-4_{\mathrm{A}}\right), 72.1\left(\mathrm{C}-3_{\mathrm{B}}\right), 72.0\left(\mathrm{C}-2_{\mathrm{A}}\right), 71.9\left(\mathrm{C}-5_{\mathrm{A}}\right), 71.5\left(\mathrm{C}-5_{\mathrm{B}}\right)$, $70.0\left(\mathrm{C}-2_{\mathrm{B}}\right), 69.7\left(\mathrm{C}-4_{\mathrm{B}}\right), 68.8\left(\mathrm{C}-6_{\mathrm{B}}\right), 62.7\left(\mathrm{C}-6_{\mathrm{A}}\right), 55.6$ $\left(\mathrm{OCH}_{3}\right), 20.8,20.7,20.6,20.5(2 \mathrm{C})\left(5 \mathrm{COCH}_{3}\right)$; ESIMS $m / z$ : $771.2[\mathrm{M}+\mathrm{Na}]^{+}$; anal. calcd for $\mathrm{C}_{36} \mathrm{H}_{44} \mathrm{O}_{17}$ (748.26): C, 57.75; H, 5.92; found: C, 57.54; H, 6.15 .

\section{Ethyl (3,4,6-tri- $O$-acetyl-2-azido-2-deoxy- $\alpha$-D-glucopyra-} nosyl)-( $1 \rightarrow 3$ )-2,4-di-O-benzyl-1-thio- $\alpha$-L-rhamnopyranoside (6): A solution of compound $3(2.4 \mathrm{~g}, 5.04 \mathrm{mmol})$ and compound $4(1.5 \mathrm{~g}, 3.86 \mathrm{mmol})$ in anhydrous $\mathrm{CH}_{2} \mathrm{Cl}_{2}(10 \mathrm{~mL})$ was cooled to $-15{ }^{\circ} \mathrm{C}$ under argon. To the cooled reaction mixture $\mathrm{HClO}_{4}-\mathrm{SiO}_{2}(50.0 \mathrm{mg})$ was added and the reaction mixture was allowed to stir at the same temperature for $1 \mathrm{~h}$. The reaction mixture was filtered through a bed of Celite ${ }^{\circledR}$ and concentrated under reduced pressure. The crude product was purified over silica gel using hexane-EtOAc (3:1) as eluant to furnish pure compound $6(2.2 \mathrm{~g}, 81 \%)$. Yellow oil; $[\alpha]_{\mathrm{D}}{ }^{25}+63.3$ (c 1.2, $\mathrm{CHCl}_{3}$ ); IR (neat): 2930, 2110, 1750, 1455, 1368, 1233, 1094, 1039, 755, $699 \mathrm{~cm}^{-1} ;{ }^{1} \mathrm{H} \mathrm{NMR}\left(600 \mathrm{MHz}, \mathrm{CDCl}_{3}\right)$

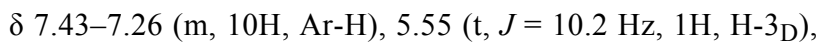
5.35 (br s, $1 \mathrm{H}, \mathrm{H}-1_{\mathrm{C}}$ ), 4.98 (t, $\left.J=10.2 \mathrm{~Hz}, 1 \mathrm{H}, \mathrm{H}-4_{\mathrm{D}}\right), 4.95$ (d, $\left.J=3.6 \mathrm{~Hz}, 1 \mathrm{H}, \mathrm{H}-1_{\mathrm{D}}\right), 4.91\left(\mathrm{~d}, J=11.4 \mathrm{~Hz}, 1 \mathrm{H}, \mathrm{PhCH}_{2}\right), 4.74$ (d, $\left.J=12.0 \mathrm{~Hz}, 1 \mathrm{H}, \mathrm{PhCH}_{2}\right), 4.70\left(\mathrm{~d}, J=11.4 \mathrm{~Hz}, 1 \mathrm{H}, \mathrm{PhCH}_{2}\right)$, $4.64\left(\mathrm{~d}, J=12.0 \mathrm{~Hz}, 1 \mathrm{H}, \mathrm{PhCH}_{2}\right), 4.15-4.13\left(\mathrm{~m}, 1 \mathrm{H}, \mathrm{H}-5_{\mathrm{D}}\right)$, $4.08-4.03\left(\mathrm{~m}, 2 \mathrm{H}, \mathrm{H}-5_{\mathrm{C}}, \mathrm{H}-6_{\mathrm{aD}}\right), 4.00$ (dd, $J=9.6,3.0 \mathrm{~Hz}, 1 \mathrm{H}$, $\mathrm{H}-3_{\mathrm{C}}$ ), 3.93 (br s, $1 \mathrm{H}, \mathrm{H}-2_{\mathrm{C}}$ ), 3.88 (d, $\left.J=12.0 \mathrm{~Hz}, 1 \mathrm{H}, \mathrm{H}-6_{\mathrm{bD}}\right)$, $3.69\left(\mathrm{t}, J=9.6 \mathrm{~Hz}, 1 \mathrm{H}, \mathrm{H}-4_{\mathrm{C}}\right), 3.31(\mathrm{dd}, J=10.8,3.6 \mathrm{~Hz}, 1 \mathrm{H}$, $\left.\mathrm{H}-2_{\mathrm{D}}\right), 2.61-2.56\left(\mathrm{~m}, 2 \mathrm{H}, \mathrm{SCH}_{2} \mathrm{CH}_{3}\right), 2.08,2.05,1.90(3 \mathrm{~s}, 9 \mathrm{H}$, $\left.3 \mathrm{COCH}_{3}\right), 1.36\left(\mathrm{~d}, J=6.0 \mathrm{~Hz}, 3 \mathrm{H}, \mathrm{CCH}_{3}\right), 1.25$ (t, $J=7.2 \mathrm{~Hz}$, $\left.3 \mathrm{H}, \mathrm{SCH}_{2} \mathrm{CH}_{3}\right) ;{ }^{13} \mathrm{C} \mathrm{NMR}\left(150 \mathrm{MHz}, \mathrm{CDCl}_{3}\right) \delta 170.5,169.8$, 
$169.6\left(3 \mathrm{COCH}_{3}\right), 138.0-127.6(\mathrm{Ar}-\mathrm{C}), 92.8\left(\mathrm{C}-1_{\mathrm{D}}\right), 81.0$ $\left(\mathrm{C}-1_{\mathrm{C}}\right), 79.4\left(\mathrm{C}-3_{\mathrm{C}}\right), 75.5\left(\mathrm{PhCH}_{2}\right), 74.6\left(\mathrm{C}-3_{\mathrm{C}}\right), 74.2\left(\mathrm{C}-2_{\mathrm{C}}\right)$, $71.8\left(\mathrm{PhCH}_{2}\right), 70.4\left(\mathrm{C}-3_{\mathrm{D}}\right), 68.4\left(\mathrm{C}-5_{\mathrm{C}}\right), 68.1\left(\mathrm{C}-4_{\mathrm{D}}\right), 67.3$ $\left(\mathrm{C}-5_{\mathrm{D}}\right), 61.5\left(\mathrm{C}-6_{\mathrm{D}}\right), 60.6\left(\mathrm{C}-2_{\mathrm{D}}\right), 25.4\left(\mathrm{SCH}_{2} \mathrm{CH}_{3}\right), 20.7,20.6$, $20.5\left(3 \mathrm{COCH}_{3}\right), 17.7\left(\mathrm{CCH}_{3}\right), 14.9\left(\mathrm{SCH}_{2} \mathrm{CH}_{3}\right)$; ESIMS $m / z$ : $724.2[\mathrm{M}+\mathrm{Na}]^{+}$; anal. calcd for $\mathrm{C}_{34} \mathrm{H}_{43} \mathrm{~N}_{3} \mathrm{O}_{11} \mathrm{~S}$ (701.26): $\mathrm{C}$, 58.19; H, 6.18; found: C, 58.0; H, 6.42 .

Ethyl (2-azido-3,4,6-tri-O-benzyl-2-deoxy- $\alpha$-D-glucopyranosyl)-( $\rightarrow 3$ )-2,4-di-O-benzyl-1-thio- $\alpha$-L-rhamnopyranoside (7): To a solution of compound $6(2.0 \mathrm{~g}, 2.85 \mathrm{mmol})$ in THF $(10 \mathrm{~mL})$ powdered $\mathrm{NaOH}(1.0 \mathrm{~g}, 25 \mathrm{mmol})$, benzyl bromide $(2.1 \mathrm{~mL}, 17.6 \mathrm{mmol})$ and $n-\mathrm{Bu}_{4} \mathrm{NBr}(50 \mathrm{mg})$ were added and the reaction mixture was allowed to stir at $\mathrm{rt}$ for $2 \mathrm{~h}$. The reaction mixture was poured into water and extracted with $\mathrm{CH}_{2} \mathrm{Cl}_{2}(100 \mathrm{~mL})$. The organic layer was washed with water, dried $\left(\mathrm{Na}_{2} \mathrm{SO}_{4}\right)$ and concentrated under reduced pressure. The crude product was purified over silica gel using hexane-EtOAc (6:1) as eluant to give pure compound 7 (2.2 g, 91\%). Yellow oil; $[\alpha]_{\mathrm{D}}{ }^{25}+12.0\left(c 1.2, \mathrm{CHCl}_{3}\right)$; IR (neat): 3430, 3031, 2925, 2107, 1642, 1496, 1454, 1361, 1215, 1091, 1051, 1028, 751, $697 \mathrm{~cm}^{-1} ;{ }^{1} \mathrm{H} \mathrm{NMR}\left(600 \mathrm{MHz}, \mathrm{CDCl}_{3}\right) \delta 7.45-7.07(\mathrm{~m}, 25 \mathrm{H}$, Ar-H), 5.32 (br s, $1 \mathrm{H}, \mathrm{H}-1_{\mathrm{C}}$ ), $4.93\left(\mathrm{~d}, J=3.6 \mathrm{~Hz}, 1 \mathrm{H}, \mathrm{H}-1_{\mathrm{D}}\right.$ ), 4.85 (br s, $2 \mathrm{H}, \mathrm{PhCH}_{2}$ ), 4.83 (d, J=10.2 Hz, 1H, $\mathrm{PhCH}_{2}$ ), 4.77 (d, $\left.J=10.8 \mathrm{~Hz}, 1 \mathrm{H}, \mathrm{PhCH}_{2}\right), 4.74$ (d, $J=12.0 \mathrm{~Hz}, 1 \mathrm{H}, \mathrm{PhCH}_{2}$ ), 4.69 (d, $\left.J=12.0 \mathrm{~Hz}, 1 \mathrm{H}, \mathrm{PhCH}_{2}\right), 4.60(\mathrm{~d}, J=12.6 \mathrm{~Hz}, 1 \mathrm{H}$, $\left.\mathrm{PhCH}_{2}\right), 4.55\left(\mathrm{~d}, J=10.2 \mathrm{~Hz}, 1 \mathrm{H}, \mathrm{PhCH}_{2}\right), 4.48(\mathrm{~d}, J=$ $10.8 \mathrm{~Hz}, 1 \mathrm{H}, \mathrm{PhCH}_{2}$ ), 4.35 (d, $\left.J=12.6 \mathrm{~Hz}, 1 \mathrm{H}, \mathrm{PhCH}_{2}\right), 4.07$ (t, $\left.J=9.6 \mathrm{~Hz}, 1 \mathrm{H}, \mathrm{H}-3_{\mathrm{D}}\right), 4.04-3.99\left(\mathrm{~m}, 3 \mathrm{H}, \mathrm{H}-3_{\mathrm{C}}, \mathrm{H}-5_{\mathrm{C}}\right.$, H- $5_{\mathrm{D}}$ ), 3.93 (br s, $1 \mathrm{H}, \mathrm{H}-2_{\mathrm{C}}$ ), 3.79 (t, $J=9.6 \mathrm{~Hz}, 1 \mathrm{H}, \mathrm{H}-4_{\mathrm{C}}$ ), $3.66\left(\mathrm{t}, J=9.6 \mathrm{~Hz}, 1 \mathrm{H}, \mathrm{H}-4_{\mathrm{D}}\right), 3.60(\mathrm{dd}, J=10.8,2.4 \mathrm{~Hz}, 1 \mathrm{H}$, $\left.\mathrm{H}-6_{\mathrm{aD}}\right), 3.51\left(\mathrm{dd}, J=10.8,1.2 \mathrm{~Hz}, 1 \mathrm{H}, \mathrm{H}-6_{\mathrm{aD}}\right), 3.40$ (dd, $J=$ 10.2, 3.6 Hz, $1 \mathrm{H}, \mathrm{H}-2 \mathrm{D}), 2.60-2.50$ (m, $\left.2 \mathrm{H}, \mathrm{SCH}_{2} \mathrm{CH}_{3}\right), 1.35$ (d, $\left.J=6.0 \mathrm{~Hz}, 3 \mathrm{H}, \mathrm{CCH}_{3}\right), 1.22\left(\mathrm{t}, J=7.8 \mathrm{~Hz}, 3 \mathrm{H}, \mathrm{SCH}_{2} \mathrm{CH}_{3}\right)$; ${ }^{13} \mathrm{C}$ NMR $\left(150 \mathrm{MHz}, \mathrm{CDCl}_{3}\right.$ ) $\delta$ 137.7-127.5 (Ar-C), 93.2 $\left(\mathrm{C}-1_{\mathrm{D}}\right), 81.1\left(\mathrm{C}-1_{\mathrm{C}}\right), 80.2\left(\mathrm{C}-3_{\mathrm{D}}\right), 79.8\left(\mathrm{C}-4_{\mathrm{D}}\right), 78.1\left(\mathrm{C}-4_{\mathrm{C}}\right)$, $75.9\left(\mathrm{PhCH}_{2}\right), 74.8\left(\mathrm{PhCH}_{2}\right), 74.5\left(\mathrm{C}-3_{\mathrm{C}}\right), 74.4\left(\mathrm{C}-2_{\mathrm{C}}\right), 73.3$ $\left(\mathrm{PhCH}_{2}\right), 71.8\left(\mathrm{PhCH}_{2}\right), 70.4\left(\mathrm{C}-5_{\mathrm{C}}\right), 68.3\left(\mathrm{C}-5_{\mathrm{D}}\right), 67.8\left(\mathrm{C}-6_{\mathrm{D}}\right)$, $63.0(\mathrm{C}-2 \mathrm{D}), 25.4\left(\mathrm{SCH}_{2} \mathrm{CH}_{3}\right), 17.8\left(\mathrm{CCH}_{3}\right), 14.9\left(\mathrm{SCH}_{2} \mathrm{CH}_{3}\right)$; ESIMS $m / z$ : $868.3[\mathrm{M}+\mathrm{Na}]^{+}$; anal. calcd for $\mathrm{C}_{49} \mathrm{H}_{55} \mathrm{~N}_{3} \mathrm{O}_{8} \mathrm{~S}$ (845.37): C, 69.56; H, 6.55; found: C, 69.33; H, 6.80.

4-Methoxyphenyl (2-azido-3,4,6-tri- $O$-benzyl-2-deoxy- $\alpha$-Dglucopyranosyl)-(1 $\rightarrow 3$ )-(2,4-di- $O$-benzyl- $\alpha$-L-rhamnopyranosyl)-(1 $\rightarrow 4)$-(2,3-di- $O$-acetyl-6-O-benzyl- $\alpha$-D-glucopyranosyl)-( $1 \rightarrow 4)$-2,3,6-tri- $O$-acetyl- $\beta$-D-glucopyranoside (8): To a solution of compound $2(1.2 \mathrm{~g}, 1.60 \mathrm{mmol})$ and compound 7 $(1.6 \mathrm{~g}, 1.89 \mathrm{mmol})$ in anhydrous $\mathrm{CH}_{2} \mathrm{Cl}_{2}(8 \mathrm{~mL}) \mathrm{MS} 4 \AA(2.0 \mathrm{~g})$ was added and the reaction mixture was cooled to $-10{ }^{\circ} \mathrm{C}$. To the cooled reaction mixture NIS (500.0 mg, $2.22 \mathrm{mmol}$ ) and $\mathrm{HClO}_{4}-\mathrm{SiO}_{2}(20.0 \mathrm{mg})$ were added and it was allowed to stir for $1 \mathrm{~h}$ at same temperature. The reaction mixture was filtered through a bed of Celite ${ }^{\circledR}$ and washed with $\mathrm{CH}_{2} \mathrm{Cl}_{2}$ $(100 \mathrm{~mL})$. The organic layer was successively washed with $5 \%$ aq. $\mathrm{Na}_{2} \mathrm{~S}_{2} \mathrm{O}_{3}, \mathrm{NaHCO}_{3}$ solution and water, dried $\left(\mathrm{Na}_{2} \mathrm{SO}_{4}\right)$ and concentrated under reduced pressure. The crude product was purified over silica gel using hexane-EtOAc (2:1) as eluant to give pure compound $8(2.0 \mathrm{~g}, 82 \%)$. White solid; $\mathrm{mp} 66-68{ }^{\circ} \mathrm{C}$; $[\alpha]_{\mathrm{D}}{ }^{25}+18.2\left(c 1.2, \mathrm{CHCl}_{3}\right)$; IR (KBr): 3428, 3032, 2932, 2108, $1753,1508,1455,1368,1230,1044,740,698 \mathrm{~cm}^{-1}$; ${ }^{1} \mathrm{H}$ NMR $\left(600 \mathrm{MHz}, \mathrm{CDCl}_{3}\right) \delta 7.35-7.05(\mathrm{~m}, 30 \mathrm{H}, \mathrm{Ar}-\mathrm{H}), 6.91(\mathrm{~d}, J=$ $9.0 \mathrm{~Hz}, 2 \mathrm{H}, \mathrm{Ar}-\mathrm{H}), 6.80(J=9.0 \mathrm{~Hz}, 2 \mathrm{H}, \mathrm{Ar}-\mathrm{H}), 5.34$ (d, $J=$ $\left.4.5 \mathrm{~Hz}, 1 \mathrm{H}, \mathrm{H}-1_{\mathrm{B}}\right), 5.29\left(\mathrm{t}, J=10.0 \mathrm{~Hz}, 1 \mathrm{H}, \mathrm{H}-3_{\mathrm{A}}\right), 5.27(\mathrm{t}, J=$ $\left.9.0 \mathrm{~Hz}, 1 \mathrm{H}, \mathrm{H}-3_{\mathrm{B}}\right), 5.04\left(\mathrm{dd}, J=8.0 \mathrm{~Hz}\right.$ each, $\left.1 \mathrm{H}, \mathrm{H}-2_{\mathrm{A}}\right), 4.97$ $\left(\mathrm{d}, J=3.5 \mathrm{~Hz}, 1 \mathrm{H}, \mathrm{H}-1_{\mathrm{D}}\right), 4.95\left(\mathrm{~d}, J=8.0 \mathrm{~Hz}, 1 \mathrm{H}, \mathrm{H}-1_{\mathrm{A}}\right), 4.89$ $\left(\mathrm{d}, J=2.0 \mathrm{~Hz}, 1 \mathrm{H}, \mathrm{H}-1_{\mathrm{C}}\right), 4.85-4.80\left(\mathrm{~m}, 2 \mathrm{H}, \mathrm{PhCH}_{2}\right)$, 4.78-4.75 (m, 3H, H-2 $\left.\mathrm{B}, \mathrm{PhCH}_{2}\right), 4.65(\mathrm{~d}, J=12.0 \mathrm{~Hz}, 1 \mathrm{H}$, $\mathrm{PhCH}_{2}$ ), 4.60 (d, $\left.J=12.0 \mathrm{~Hz}, 1 \mathrm{H}, \mathrm{PhCH}_{2}\right), 4.58-4.44$ (m, 5H, $\left.\mathrm{PhCH}_{2}\right), 4.41\left(\mathrm{dd}, J=12.5,2.5 \mathrm{~Hz}, 1 \mathrm{H}, \mathrm{H}-6_{\mathrm{aA}}\right), 4.35(\mathrm{~d}, J=$ $\left.12.0 \mathrm{~Hz}, 1 \mathrm{H}, \mathrm{PhCH}_{2}\right), 4.21$ (dd, $J=12.5,2.5 \mathrm{~Hz}, 1 \mathrm{H}, \mathrm{H}-6_{\mathrm{bA}}$ ), 4.05-4.01 (2 t, $J=9.5 \mathrm{~Hz}$ each, $\left.2 \mathrm{H}, \mathrm{H}-3_{\mathrm{D}}, \mathrm{H}-4_{\mathrm{C}}\right), 3.98-3.92$ (m, $2 \mathrm{H}, \mathrm{H}-3 \mathrm{c}, \mathrm{H}-5 \mathrm{c}), 3.90$ (t, $\left.J=10.0 \mathrm{~Hz}, 1 \mathrm{H}, \mathrm{H}-4_{\mathrm{D}}\right), 3.81$ (t, $J=$ $\left.8.5 \mathrm{~Hz}, 1 \mathrm{H}, \mathrm{H}-4_{\mathrm{A}}\right), 3.77\left(\mathrm{~s}, 3 \mathrm{H}, \mathrm{OCH}_{3}\right), 3.76-3.74(\mathrm{~m}, 1 \mathrm{H}$, $\left.\mathrm{H}-4_{\mathrm{B}}\right), 3.72-3.71\left(\mathrm{~m}, 1 \mathrm{H}, \mathrm{H}-5_{\mathrm{B}}\right), 3.69-3.65\left(\mathrm{~m}, 2 \mathrm{H}, \mathrm{H}-5_{\mathrm{A}}\right.$, $\left.\mathrm{H}-6_{\mathrm{aB}}\right), 3.63-3.61\left(\mathrm{~m}, 1 \mathrm{H}, \mathrm{H}-6_{\mathrm{aD}}\right), 3.58-3.55\left(\mathrm{~m}, 3 \mathrm{H}, \mathrm{H}-2_{\mathrm{C}}\right.$, $\left.\mathrm{H}-5_{\mathrm{D}}, \mathrm{H}-6_{\mathrm{bB}}\right), 3.53-3.51$ (m, $\left.1 \mathrm{H}, \mathrm{H}-6_{\mathrm{bD}}\right), 3.43$ (dd, $J=10.0,3.5$ $\left.\mathrm{Hz}, 1 \mathrm{H}, \mathrm{H}-2_{\mathrm{D}}\right), 2.04,2.02,2.01,1.99,1.95(5 \mathrm{~s}, 15 \mathrm{H}$, $\left.5 \mathrm{COCH}_{3}\right), 1.25\left(\mathrm{~d}, J=6.2 \mathrm{~Hz}, 3 \mathrm{H}, \mathrm{CCH}_{3}\right) ;{ }^{13} \mathrm{C} \mathrm{NMR}$ $\left(150 \mathrm{MHz}, \mathrm{CDCl}_{3}\right) \delta 170.7,170.2(2 \mathrm{C}), 169.8,169.7$ $\left(5 \mathrm{COCH}_{3}\right), 155.7-114.5(\mathrm{Ar}-\mathrm{C}), 99.7\left(\mathrm{C}-1_{\mathrm{A}}\right), 98.8\left(\mathrm{C}-1_{\mathrm{C}}\right), 96.0$ $\left(\mathrm{C}-1_{\mathrm{B}}\right), 93.9\left(\mathrm{C}-1_{\mathrm{D}}\right), 80.2\left(\mathrm{C}-3_{\mathrm{D}}\right), 79.4\left(\mathrm{C}-4_{\mathrm{D}}\right), 78.2\left(\mathrm{C}-5_{\mathrm{A}}\right)$, $77.2\left(\mathrm{C}-5_{\mathrm{B}}\right), 75.5\left(\mathrm{PhCH}_{2}\right), 75.4\left(\mathrm{C}-3_{\mathrm{A}}\right), 75.3\left(\mathrm{PhCH}_{2}\right), 74.8$ $\left(\mathrm{PhCH}_{2}\right), 74.3\left(\mathrm{C}-2_{\mathrm{B}}\right), 74.2\left(\mathrm{C}-4_{\mathrm{C}}\right), 73.7\left(\mathrm{PhCH}_{2}\right), 73.3$ $\left(\mathrm{PhCH}_{2}\right), 72.6\left(2 \mathrm{C}, \mathrm{C}-5_{\mathrm{D}}, \mathrm{PhCH}_{2}\right), 72.2\left(\mathrm{C}-4_{\mathrm{A}}\right), 72.1\left(\mathrm{C}-2_{\mathrm{A}}\right)$, $71.3\left(\mathrm{C}-4_{\mathrm{B}}\right), 70.7\left(2 \mathrm{C}, \mathrm{C}-3_{\mathrm{C}}, \mathrm{C}-5_{\mathrm{C}}\right), 70.4\left(\mathrm{C}-3_{\mathrm{B}}\right), 69.0\left(\mathrm{C}-2_{\mathrm{C}}\right)$, $67.9\left(\mathrm{C}-6_{\mathrm{B}}\right), 67.8\left(\mathrm{C}-6_{\mathrm{D}}\right), 63.3\left(\mathrm{C}-2_{\mathrm{D}}\right), 62.6\left(\mathrm{C}-6_{\mathrm{A}}\right), 55.6$ $\left(\mathrm{OCH}_{3}\right), 21.0,20.9,20.7,20.6(2 \mathrm{C})\left(5 \mathrm{COCH}_{3}\right), 17.9\left(\mathrm{CCH}_{3}\right)$; ESIMS $m / z$ : $1554.6[\mathrm{M}+\mathrm{Na}]^{+}$; anal. calcd for $\mathrm{C}_{83} \mathrm{H}_{93} \mathrm{~N}_{3} \mathrm{O}_{25}$ (1531.61): C, 65.04; H, 6.12; found: C, 64.82; H, 6.36.

\section{4-Methoxyphenyl (2-acetamido-2-deoxy-a-D-glucopyra-} nosyl)-( $1 \rightarrow 3)$-( $\alpha$-L-rhamnopyranosyl)-( $1 \rightarrow 4)$-( $\alpha$-D-glucopyranosyl)-(1 $\rightarrow$ 4)-sodium $\beta$-D-glucopyranosid uronate (1): A solution of compound $8(1.3 \mathrm{~g}, 0.85 \mathrm{mmol})$ in $0.1 \mathrm{M} \mathrm{CH}_{3} \mathrm{ONa}$ in $\mathrm{CH}_{3} \mathrm{OH}(25 \mathrm{~mL})$ was allowed to stir at $\mathrm{rt}$ for $3 \mathrm{~h}$ and neutralized with Dowex 50W X8 $\left(\mathrm{H}^{+}\right)$resin. The reaction mixture was filtered and concentrated under reduced pressure. To a solution of the crude product in $\mathrm{CH}_{2} \mathrm{Cl}_{2}(25 \mathrm{~mL})$ and $\mathrm{H}_{2} \mathrm{O}(4 \mathrm{~mL})$ were sequentially added aq. $\mathrm{NaBr}(2 \mathrm{~mL}, 1 \mathrm{M})$, aq. TBAB $(2.5 \mathrm{~mL}$, $1 \mathrm{M})$, TEMPO (100.0 mg, $0.64 \mathrm{mmol})$, satd. $\mathrm{NaHCO}_{3}$ solution $(10 \mathrm{~mL})$ and $4 \%$ aq. $\mathrm{NaOCl}(15 \mathrm{~mL})$ were added and the reaction mixture was allowed to stir at $0-5{ }^{\circ} \mathrm{C}$ for $2 \mathrm{~h}$. The reaction 
mixture was neutralized with $1 \mathrm{~N}$ aq. $\mathrm{HCl}$. To the reaction mixture tert-butanol $(25 \mathrm{~mL}), 2$-methylbut-2-ene $(20 \mathrm{~mL}, 2 \mathrm{M}$ solution in THF), aq. $\mathrm{NaClO}_{2}(1.5 \mathrm{~g}$ in $5 \mathrm{~mL})$ and aq. $\mathrm{NaH}_{2} \mathrm{PO}_{4}$ $(1.5 \mathrm{~g}$ in $5 \mathrm{~mL})$ were added and the reaction mixture was stirred at room temperature for $3 \mathrm{~h}$. The reaction mixture was diluted with satd. aq. $\mathrm{NaH}_{2} \mathrm{PO}_{4}$ and extracted with $\mathrm{CH}_{2} \mathrm{Cl}_{2}(150 \mathrm{~mL})$. The organic layer was washed with water, dried $\left(\mathrm{Na}_{2} \mathrm{SO}_{4}\right)$ and concentrated to dryness. The crude product was passed through a short pad of silica gel using EtOAc-toluene (2:1) as eluant. To a solution of the oxidized product $(800.0 \mathrm{mg})$ in $\mathrm{CH}_{3} \mathrm{OH}$-EtOAc $(20 \mathrm{~mL}, 10: 1 \mathrm{v} / v) 20 \% \mathrm{Pd}(\mathrm{OH})_{2} / \mathrm{C}(150.0 \mathrm{mg})$ was added and the reaction mixture was allowed to stir at room temperature under a positive pressure of hydrogen for $10 \mathrm{~h}$. The reaction mixture was filtered through a bed of Celite ${ }^{\circledR}$ and evaporated to dryness. To a solution of the crude product in $\mathrm{CH}_{3} \mathrm{OH}$ $(10 \mathrm{~mL})$ acetic anhydride $(2 \mathrm{~mL})$ was added and the solution was kept at $\mathrm{rt}$ for $30 \mathrm{~min}$. The solvents were removed under reduced pressure and the product was passed through a Sephadex ${ }^{\circledR}$ LH-20 column using $\mathrm{CH}_{3} \mathrm{OH}-\mathrm{H}_{2} \mathrm{O}(60 \mathrm{~mL}, 4: 1 \mathrm{v} / \mathrm{v})$ as eluant to give pure compound $\mathbf{1}(450.0 \mathrm{mg}, 64 \%)$. White powder; $[\alpha]_{\mathrm{D}}{ }^{25}+14\left(c\right.$ 1.0, $\left.\mathrm{CH}_{3} \mathrm{OH}\right)$; IR (KBr): 3428, 2937, $1621,1366,1152,1087,669 \mathrm{~cm}^{-1} ;{ }^{1} \mathrm{H}$ NMR $(600 \mathrm{MHz}$, $\left.\mathrm{CD}_{3} \mathrm{OD}\right) \delta 7.04(\mathrm{~d}, J=9.0 \mathrm{~Hz}, 2 \mathrm{H}, \mathrm{Ar}-\mathrm{H}), 6.84(\mathrm{~d}, J=9.0 \mathrm{~Hz}$, $2 \mathrm{H}, \operatorname{Ar}-\mathrm{H}), 5.20\left(\mathrm{~d}, J=3.6 \mathrm{~Hz}, 1 \mathrm{H}, \mathrm{H}-1_{\mathrm{B}}\right), 4.93$ (br s, $1 \mathrm{H}$, $\left.\mathrm{H}-1_{\mathrm{D}}\right), 4.89$ (br s, $\left.1 \mathrm{H}, \mathrm{H}-1_{\mathrm{C}}\right), 4.82\left(\mathrm{~d}, J=7.8 \mathrm{~Hz}, 1 \mathrm{H}, \mathrm{H}-1_{\mathrm{A}}\right)$, 4.03-3.98 (m, $\left.2 \mathrm{H}, \mathrm{H}-2_{\mathrm{A}}, \mathrm{H}-5_{\mathrm{C}}\right), 3.97-3.95$ (m, $2 \mathrm{H}, \mathrm{H}-2_{\mathrm{C}}$, $\left.\mathrm{H}-2_{\mathrm{D}}\right), 3.88-3.86\left(\mathrm{~m}, 1 \mathrm{H}, \mathrm{H}-6_{\mathrm{aD}}\right), 3.83-3.75\left(\mathrm{~m}, 5 \mathrm{H}, \mathrm{H}-3_{\mathrm{A}}\right.$, $\left.\mathrm{H}-3_{\mathrm{B}}, \mathrm{H}-5_{\mathrm{D}}, \mathrm{H}-6_{\mathrm{aB}}, \mathrm{H}-6_{\mathrm{bD}}\right), 3.74$ (s, 3H, OCH $), 3.73-3.71$ (m, $\left.1 \mathrm{H}, \mathrm{H}-4_{\mathrm{A}}\right), 3.70-3.67\left(\mathrm{~m}, 2 \mathrm{H}, \mathrm{H}-2_{\mathrm{B}}, \mathrm{H}-6_{\mathrm{bB}}\right), 3.64(\mathrm{t}, J=9.6 \mathrm{~Hz}$, $\left.1 \mathrm{H}, \mathrm{H}-3_{\mathrm{D}}\right), 3.56-3.50\left(\mathrm{~m}, 4 \mathrm{H}, \mathrm{H}-4_{\mathrm{B}}, \mathrm{H}-4_{\mathrm{C}}, \mathrm{H}-5_{\mathrm{A}}, \mathrm{H}-5_{\mathrm{B}}\right)$, 3.49-3.44 (m, 2H, H-3, H-4 $), 2.02$ (s, 3H, $\mathrm{COCH}_{3}$ ), 1.30 (d, $\left.J=6.1 \mathrm{~Hz}, 3 \mathrm{H}, \mathrm{CCH}_{3}\right) ;{ }^{13} \mathrm{C} \mathrm{NMR}\left(150 \mathrm{MHz}, \mathrm{CDCl}_{3}\right) \delta 175.0$ $(\mathrm{COONa}), 172.6\left(\mathrm{NHCOCH}_{3}\right), 156.7-115.5(\mathrm{Ar}-\mathrm{C}), 103.3$ $\left(\mathrm{C}-1_{\mathrm{A}}\right), 102.8\left(\mathrm{C}-1_{\mathrm{B}}\right), 102.4\left(\mathrm{C}-1_{\mathrm{C}}\right), 96.7\left(\mathrm{C}-1_{\mathrm{D}}\right), 81.2\left(\mathrm{C}-3_{\mathrm{D}}\right)$, $79.5\left(\mathrm{C}-4_{\mathrm{B}}\right), 78.1\left(\mathrm{C}-3_{\mathrm{A}}\right), 77.7\left(\mathrm{C}-4_{\mathrm{A}}\right), 76.6\left(\mathrm{C}-5_{\mathrm{A}}\right), 74.6$ $\left(\mathrm{C}-3_{\mathrm{C}}\right), 74.2\left(\mathrm{C}-4_{\mathrm{C}}\right), 73.8\left(\mathrm{C}-2_{\mathrm{B}}\right), 73.6\left(\mathrm{C}-2_{\mathrm{A}}\right), 73.0\left(\mathrm{C}-5_{\mathrm{D}}\right)$, $72.0\left(\mathrm{C}-5_{\mathrm{B}}\right), 71.9\left(\mathrm{C}-4_{\mathrm{D}}\right), 70.8\left(\mathrm{C}-5_{\mathrm{C}}\right), 69.3\left(\mathrm{C}-2_{\mathrm{C}}\right), 62.1$ $\left(\mathrm{C}-6_{\mathrm{B}}\right), 62.0\left(\mathrm{C}-6_{\mathrm{D}}\right), 56.2\left(\mathrm{OCH}_{3}\right), 55.3\left(\mathrm{C}-2_{\mathrm{D}}\right), 23.1$ $\left(\mathrm{NHCOCH}_{3}\right), 18.1\left(\mathrm{CCH}_{3}\right)$; ESIMS $m / z: 834.2[\mathrm{M}+\mathrm{H}]^{+}$; anal. calcd for $\mathrm{C}_{33} \mathrm{H}_{48} \mathrm{NNaO}_{22}$ (833.26): C, 47.54; $\mathrm{H}, 5.80$; found: $\mathrm{C}$, 47.72; H, 6.07 .

\section{Supporting Information}

\section{Supporting Information File 1}

1D and 2D NMR spectra of compounds $2,6,7,8$ and 1.

[http://www.beilstein-journals.org/bjoc/content/

supplementary/1860-5397-7-137-S1.pdf]

\section{Acknowledgements}

A. S. thanks CSIR, New Delhi for providing a Senior Research Fellowship. This work was supported by Bose Institute, Kolkata.

\section{References}

1. von Seidlein, L.; Kim, D. R.; Ali, M.; Lee, H.; Wang, X.; Thiem, V. D.; Canh, D. G.; Chaicumpa, W.; Agtini, M. D.; Hossain, A.; Bhutta, Z. A.; Mason, C.; Sethabutr, O.; Talukder, K.; Nair, G. B.; Deen, J. L.; Kotloff, K.; Clemens, J. PLoS Med. 2006, 1556-1569.

2. Wachsmuth, K.; Morris, G. K. In Shigella, Foodborne Bacterial Pathogens; Doyle, M. P., Ed.; Marcel Dekker Inc: New York, 1989; pp 447-462.

3. Gupta, A.; Polyak, C. S.; Bishop, R. D.; Sobel, J.; Mintz, E. D. Clin. Infect. Dis. 2004, 38, 1372-1377. doi:10.1086/386326

4. Liu, B.; Knirel, Y. A.; Feng, L.; Perepelov, A. V.; Senchenkova, S. N.; Wang, Q.; Reeves, P. R.; Wang, L. FEMS Microbiol. Rev. 2008, 32, 627-653. doi:10.1111/j.1574-6976.2008.00114.x

5. Wathen-Grady, H. G.; Davis, B. R.; Morris, G. K. J. Clin. Microbiol. 1985, 21, 129-132.

6. Reeves, P. R.; Wang, L. Curr. Top. Microbiol. Immunol. 2002, 264, 109-135.

7. Knirel, Y. A.; Kochetkov, N. K. Biochemistry (Moscow) 1994, 59, 1325-1383.

8. L'vov, V. L.; Musina, L. I.; Shashkov, A. S.; Ermakov, G. P.; Dmitriev, B. A. Bioorg. Khim. 1987, 13, 1245-1255.

9. Kosek, M.; Yori, P. P.; Olortegui, M. P. Curr. Opin. Infect. Dis. 2010, 23, 475-480. doi:10.1097/QCO.0b013e32833da204

10. Bhan, M. K.; Raj, P.; Srivastava, R.; Bhandari, N.; Kumar, R. Indian Pediatr. 1988, 25, 804-805.

11. Klee, S. R.; Tzschaschel, B. D.; Singh, M.; Falt, I.; Lindberg, A. A.; Timmis, K. N.; Guzman, C. A. Microb. Pathog. 1997, 22, 363-376. doi:10.1006/mpat.1996.0127

12. Kweon, M.-N. Curr. Opin. Infect. Dis. 2008, 21, 313-318. doi:10.1097/QCO.0b013e3282f88b92

13. Niyogi, S. K. J. Microbiol. 2005, 43, 133-143.

14. Oaks, E. V.; Turbyfill, K. R. Vaccine 2006, 24, 2290-2301. doi:10.1016/j.vaccine.2005.11.040

15. Pozsgay, V.; Kubler-Kielb, J. ACS Symposium Ser. 2007, 960, 238-252. doi:10.1021/bk-2007-0960.ch014

16. Phalipon, A.; Costachel, C.; Grandjean, C.; Thuizat, A.; Guerreiro, C.; Tanguy, M.; Nato, F.; Vulliez-Le Normand, B.; Belot, F.; Wright, K.; Marcel-Peyre, V.; Sansonetti, P. J.; Mulard, L. A. J. Immunol. 2006, 176, 1686-1694.

17. Pozsgay, V.; Coxon, B.; Glaudemans, C. P. J.; Schneerson, R.; Robbins, J. B. Synlett 2003, 743-767. doi:10.1055/s-2003-38724

18. Pozsgay, V. Angew. Chem., Int. Ed. 1998, 37, 138-142. doi:10.1002/(SICI)1521-3773(19980202)37:1/2<138::AID-ANIE138>3.0 .CO;2-T

19. Carlin, N. I.; Bundle, D. R.; Lindberg, A. A. J. Immunol. 1987, 138, $4419-4427$.

20. Santra, A.; Guchhait, G.; Misra, A. K. Green Chem. 2011, 13, 1345-1351. doi:10.1039/c1gc15122c

21. Panchadhayee, R.; Misra, A. K. Synlett 2010, 1193-1196. doi:10.1055/s-0029-1219798

22. Madhusudan, S. K.; Agnihotri, G.; Negi, D. S.; Misra, A. K. Carbohydr. Res. 2005, 340, 1373-1377. doi:10.1016/j.carres.2005.03.007 
23. Ludek, O. R.; Gu, W.; Gildersleeve, J. C. Carbohydr. Res. 2010, 345, 2074-2078. doi:10.1016/j.carres.2010.07.030

24. Du, Y.; Wei, G.; Cheng, S.; Hua, Y.; Linhardt, R. J. Tetrahedron Lett. 2006, 47, 307-310. doi:10.1016/j.tetlet.2005.11.025

25. Mukherjee, C.; Misra, A. K. Synthesis 2007, 683-692. doi:10.1055/s-2007-965913

26. Mukhopadhyay, B.; Collet, B.; Field, R. A. Tetrahedron Lett. 2005, 46, 5923-5925. doi:10.1016/j.tetlet.2005.06.119

27. Huang, L.; Teumelsan, N.; Huang, X. Chem.-Eur. J. 2006, 12, 5246-5252. doi:10.1002/chem.200600290

28. Mukherjee, C.; Misra, A. K. Glycoconjugate J. 2008, 25, 111-119. doi:10.1007/s10719-007-9061-0

29. Panchadhayee, R.; Misra, A. K. Tetrahedron: Asymmetry 2009, 20 1550-1555. doi:10.1016/j.tetasy.2009.05.026

30. Attolino, E.; Rising, T. W. D. F.; Heidecke, C. D.; Fairbanks, A. J. Tetrahedron: Asymmetry 2007, 18, 1721-1734. doi:10.1016/j.tetasy.2007.06.026

31. Werz, D. B.; Seeberger, P. H. Angew. Chem., Int. Ed. 2005, 44, 6315-6318. doi:10.1002/anie.200502615

32. Marmuse, L.; Nepogodiev, S. A.; Field, R. A. Tetrahedron: Asymmetry 2005, 16, 477-485. doi:10.1016/j.tetasy.2004.11.047

33. Grundler, G.; Schmidt, R. R. Liebigs Ann. Chem. 1984, 1826-1847. doi:10.1002/jlac.198419841108

34. Sajtos, F.; Hajko, J.; Kover, K. E.; Liptak, A. Carbohydr. Res. 2001, 334, 253-259. doi:10.1016/S0008-6215(01)00196-3

35. Schmidt, R. R. Angew. Chem., Int. Ed. Engl. 1986, 25, 212-235 doi:10.1002/anie.198602121

36. Bock, K.; Pedersen, C. Acta Chem. Scand., Ser. B 1975, 29, 258-264. doi:10.3891/acta.chem.scand.29b-0181

37. Duus, J. O.; Gotfredsen, C. H.; Bock, K. Chem. Rev. 2000, 100, 4589-4614. doi:10.1021/cr990302n

38. Crich, D.; Li, H. J. Org. Chem. 2002, 67, 4640-4646. doi:10.1021/jo0108818

39. Chakraborti, A. K.; Gulhane, R. Chem. Commun. 2003, 1896-1897. doi:10.1039/b304178f

\section{License and Terms}

This is an Open Access article under the terms of the Creative Commons Attribution License (http://creativecommons.org/licenses/by/2.0), which permits unrestricted use, distribution, and reproduction in any medium, provided the original work is properly cited.

The license is subject to the Beilstein Journal of Organic Chemistry terms and conditions:

(http://www.beilstein-journals.org/bjoc)

The definitive version of this article is the electronic one which can be found at: $\underline{\text { doi: } 10.3762 / \text { bjoc. } 7.137}$ 